\begin{tabular}{|l|l|l|l|l|l}
\hline Senckenbergiana lethaea & 82 & (1) & $23-34$ & 9 Fig. & Frankfurt am Main, 30.6.2002 \\
\hline
\end{tabular}

Concepts of Functional, Engineering and Constructional Morphology*

\title{
DinoMorph: Parametric Modeling of Skeletal Structures
}

\author{
With 9 Figures
}

Kent A. STEVEns

\begin{abstract}
A parametric approach towards modeling is advocated for skeletal reconstructions. Three-dimensional digital reconstructions are compared with conventional two-dimensional illustrations, particularly silhouette drawings. The advantages of the parametric system provided by the DinoMorph ${ }^{\mathrm{TM}}$ software include: open access to all data comprising the model and the rendering algorithms for the independent verification of reconstructions, tools for parametrically editing and manipulating pose, bone, and joint geometry, visualization of assemblies in three-dimensions from arbitrary perspectives, multiple resolution models of skeletal element morphology (from schematic to highly detailed), and extensibility to support specific research objectives. The system architecture and current capabilities are described and illustrated.
\end{abstract}

Key words: biomechanics, simulation, sauropod dinosaurs, modeling, 3D graphics

\section{Introduction}

A model is an abstraction, a simplification, and often a scientifically useful description of a physical object or system. Plaster casts and three-dimensional digital scan of a fossil bone pertain to the specific individual, and embody the distortions, imperfections, and idiosyncrasies of the original. They are more replicas than models. In contrast, a model governed by numerical parameters is an abstraction of the object being modeled, and provides more of a description, but usually at the expense of realism. To introduce parametric modeling as it applies to skeletal reconstructions, consider first a parametric model of a specific bone, then that of an articulated skeleton.

\section{Parametric Models as a Basis for Quantitative Scientific Descriptions}

The conventional scientific description of a femur, for example, would include its measured dimensions, and a characterization of the shape of the proximal head, distal condyles, and so forth, usually accompanied with illustrations. The descrip- tion is primarily qualitative, and relies on the reader sharing a common understanding of terms such as 'pronounced', 'relatively flattened', and 'moderately well developed'. A quantitative description would also be possible if these descriptive terms could have associated numerical values. While few such quantities are readily measured, other than dimensions, they can be quantified indirectly by means of parametric modeling. A parametric model of a femur would represent topographic features such as condyles, trochanters, and so forth, with their position, magnitude, curvature and other characteristics governed by adjustable parameters. These parameters would be adjusted in the process of creating a model that resembles the original. The model can be made to resemble a specific femur, except for its idiosyncratic details and distortions not captured by the model (which are dutifully replicated by a digital scan). Parametric modeling is not intended to precisely replicate the femur's morphology, but to facilitate creating any of a range of variations on femur shape simply by adjustment of parameters. Likewise, a parametric model for the ilium would embody

*) Special issue of Senckenbergiana lethaea, edited by MichaEL Gudo, MAthias GuTMANN \& JOACHIM SCHOLZ 
parameters for quantifying the dimensions and geometric features characteristic of ilia. A parametric model can be thought of as a 'shape generator' for a class of shapes (e.g. femur or ilium). This is fundamentally distinct from the conventional digitization of a femur or ilium, for which the surface morphology and topographic features are implicit in the two-dimensional distribution of surface points. The parametric modeling paradigm extends to creating a 'skeleton generator'

To be amenable to a discrete parametric approach, the objects to be modeled must vary along a manageably small number of dimensions or attributes. The tetrapod skeleton exhibits a conservative design consisting of an axial skeleton differentiated into four vertebral regions, plus an appendicular skeleton of pectoral and pelvic girdles and associated appendages. Within the axial and appendicular skeletons, the type of elements, their number, their topological arrangement, and their articulations recur with little variation across the tetrapods. These regularities permit a broad range of taxonomic variation in skeletal design to be captured parametrically by a manageably small number of parameters. For those elements that vary in number across taxa (e.g the vertebrae in different regions, or the phalangeal formulae), specifying these counts establishes the overall body plan. Geometric parameters would then establish the proportions of each element. Bone morphology might then be depicted variously, as simple line segments or cylinders (text-fig. 1), or parametrically for each bone type as just described, or as digital replicas. Morphology is then treated like a garment that may be worn by, or removed from, the underlying framework. Likewise joints might be given a range of visual representations. Joints differ in the motion they permit, from single-axis motion (such as found in ginglymus or hinge joints), to three-axis motion (e.g. within the glenoid and acetabulum). The parametric representation of a joint begins with specifying its location relative to the associated bones proximal and distal to that joint, which establishes a basic separation appropriate for the cartilage presumed to be associated with that joint. About the joint are arrayed from one to three axes of rotation (depending on the type of joint), with parameters specifying the position and direction of each. Rotation about these axes approximates joint deflection when the precise relative movement of individual joint facets need not be visualized. The articulation facets may be represented for more detailed studies of joint biomechanics.

Computer aided design (CAD) software such as Alias/ Wavefront Studio ${ }^{\mathrm{TM}}$ and 3D-Studio $\mathrm{Max}^{\mathrm{TM}}$ have been used to model hindlimb movement (GATESY, MidDlETON, JENKINS, \& SCHUBIN, 1999) and even to reconstruct an entire digitized Triceratops skeleton (CHAPMAN, ANDERSEN \& JABO, 1999; WALTERS, ChapMAn, SNYDER \& MOHN, 2000). The commercial software allows an expert user to define an assembly of 3D objects articulated by pin or ball-and-socket joints; a limb or an entire skeleton model can be constructed and animated by the same software used to design an aircraft's landing gear and visualize its extension and retraction.

Creating a biomechanical model with CAD tools requires specialized working knowledge of the commercial software plus a protracted, concerted effort to design a satisfactorily articulated skeletal assembly of joints and bones. The effort and expertise is not shared readily between laboratories, nor even between successive modeling projects within a laboratory. The creation of a skeletal model would require far less expertise and effort if the software incorporated a parametric framework, a 'skeleton generator' that reduces the modeling task to one of setting parameters to match the dimensions and shapes of a given specimen, a far easier task than having the specimen scanned and the data edited and converted to the required graphical format. Furthermore, the parametric data associated with different such models are commensurate, allowing direct quantitative comparison and even three-dimensional 'morphing' (interpolation) between a pair of such models. The volume of data required to specify a parametric model
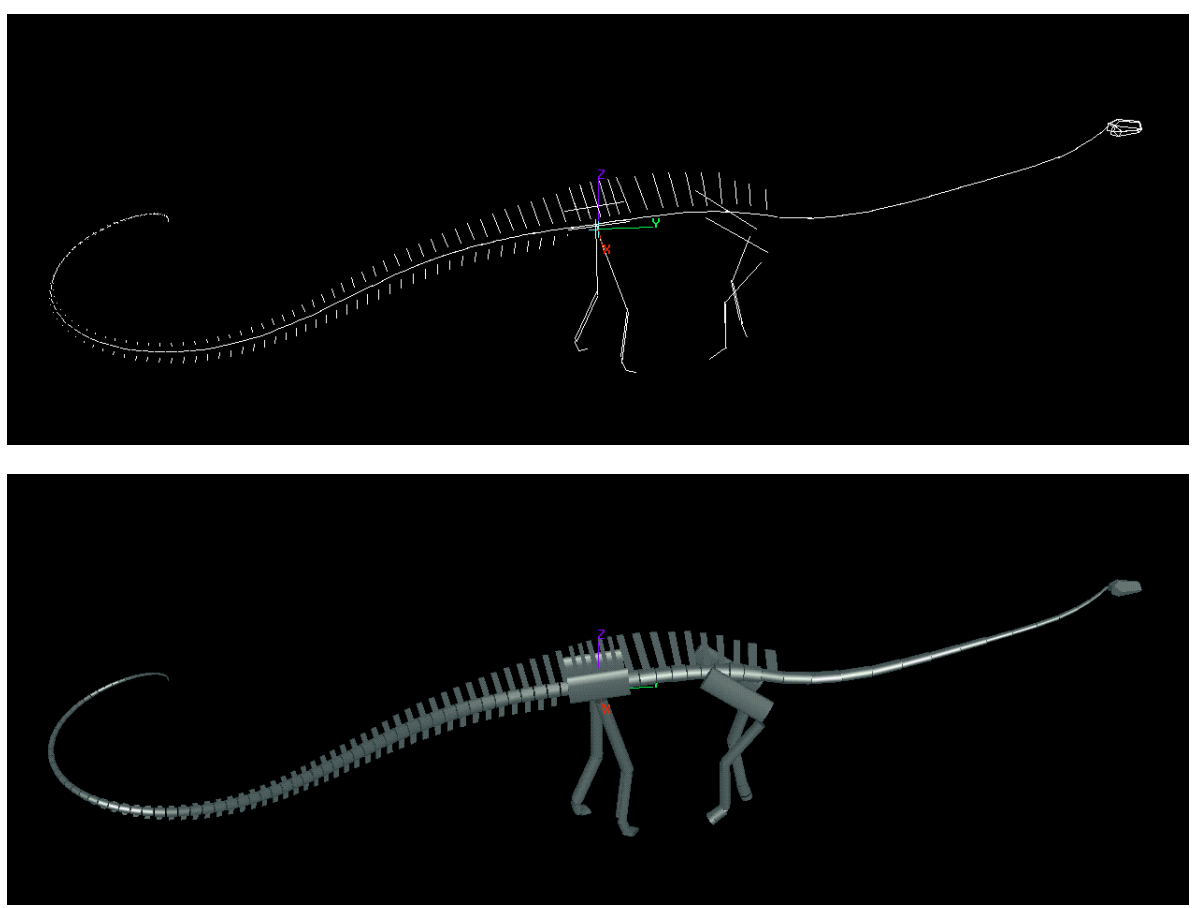

Text-fig. 1. A stick figure (a) demonstrates how the mere depiction of the length and angulation of major skeletal elements can convey overall body plan, in this case of the sauropod dinosaur Diplodocus carnegii. Rendering the same structure with solid cylinders $(b)$ provides additional information about the dimensions of the skeletal elements as well as their spatial arrangement. The bone and joint morphology can then be elaborated as needed in support of a given investigation. 
is minute, many orders of magnitude smaller than that conventionally required to form an assembly of digital replicas, facilitating the global sharing and visualization of digital reconstructions. Fortunately, these benefits of parametric modeling extend equally to modeling skeletal assemblies and the individual elements.

In reconstructing a skeleton, particularly for an extinct animal based on an incomplete specimen, there is an issue of the appropriate precision and complexity to seek for the model. Limb bone length is usually the most reliable single parameter associated with a skeletal element. The bone's overall proportions can then be estimated based on the robustness or gracility of the overall skeleton. But a more specific restoration of the bone's geometry would require introducing conjectural morphological detail. While it is conventional to create detailed yet hypothetical reconstructions of missing elements, an alternative is to represent missing elements less realistically and more symbolically. On the other hand, if a bone is available and well enough preserved to warrant high-resolution digital scanning, its morphology can be depicted quite accurately. Digitized data will always be more expensive and difficult to acquire, however, than that extracted traditionally by calipers and recorded manually, either from the original specimen or indirectly from an illustration. Furthermore, postdepositional distortion and damage frequently precludes placing them (or their digitized models) into proper articulation. They can serve, however, as a source for creating an idealized, undistorted, model.

For undistorted specimens, digitizations might seem the preferred means to represent bone morphology, but for their computational cost. At present, at least, computer graphics hardware is incapable of displaying an animated skeleton at such resolution. To work within available rendering bandwidth, usually the digitizations are 'decimated' to greatly reduce their surface complexity, and additional sculpting effort is then required to minimize their resembling crumpled paper bags or origami. A parametric model provides an alternative: some of the elements of an assembly might be modeled with high resolution when desired and the data is available, while the remaining elements would be modeled parametrically, and very simplified yet representative. This would allow the limited graphics bandwidth to be concentrated where it is most needed. By so doing, the best aspects of both modeling paradigms, the replicative and the parametric, can be combined, allowing one to import detailed morphology data, or to resort to a far more simplistic representation if such data is not available.

\section{Parametric Descriptions Facilitate Independent Evaluation of Reconstructions}

Published descriptions are traditionally illustrated with twodimensional (2D) artwork and photographic illustrations of the elements, plus an artistic reconstruction of the whole skeleton when the specimen is sufficiently complete as to permit. Animated, three-dimensional (3D) models now permit more vivid and more scientifically useful reconstructions than previously possible. Furthermore, the underlying data may also be published electronically, providing explicit access to the reconstruction's underlying dimensions, spacings, joint angles and other parameters.
Subtle yet significant dimensional inaccuracies can arise in artwork, both in the rendering of the elements themselves and in their placement - discrepancies that are difficult to uncover later (see below). Even if the individual elements are portrayed accurately, an illustration can depict but one pose, and but one hypothesis for the overall conformation of the skeleton. Neither artwork nor physical mounts are well suited to understanding the subtle consequences of local postural or positional adjustments to the overall conformation of the skeleton. A 3D model, on the other hand, provides greater access to the assumptions and subjectivity underlying any reconstruction. For example, consider the reconstruction of the pectoral girdles.

The placement of the pectoral girdles is of central importance to the reconstruction of the entire skeleton, for the height and orientation of the anterior vertebral column pivots about the acetabulum in response to changing elevation at the pectoral girdles. For instance, the low placement of the pectoral girdles in the mounted Diplodocus skeleton at the Senckenberg Museum in Frankfurt results in a high browser interpretation, dramatically different from than originally proposed (HoLLAND, 1906).

The scapulae, coracoids and sternal plates form a girdle about the ribcage, but their placement and the shape of the underlying ribcage and the curvature of the backbone has been given widely differing interpretations. Starting with the dorsal vertebral column, reconstructions range from virtually flat to highly arched. The dorsal ribs are variously interpreted as roughly perpendicular to the axis of the centra or swept back steeply, affecting the reconstructed width and curvature of the ribcage upon which the scapulae would lie, and indirectly affect the reconstruction of the girdles and pose of the forelimbs (PAUL \& CHRISTIANSEN, 2000). The scapulae would presumably be positioned such that their elongate blades conform to the curvature of the ribcage, the medial margins of the coracoids would be separated by some hypothesized distance, and the glenoids oriented in a manner consistent with accepting the proximal heads of the humeri, for a given reconstruction of the forelimbs. Other factors might enter into consideration, such as the separation between the cervical ribs of the most caudal of the cervical vertebrae, and the hypothesized location of the sternal plates.

The global conformation of a skeleton is the result of many simultaneous constraints, and adjustment of one parameter can have a ripple effect which is far easier dealt with in a digital model than a physical model. Furthermore, interpretations that differ simultaneously on many parameters (e.g. intervertebral separation, curvature to the dorsal column, and limb stance) can be compared within a common osteological framework, with confidence that aspects of the reconstruction that are presumed constant across interpretations remain so. Moreover, a parametric digital modeling system removes many of the obstacles in attempting to compare a physical mount with a $2 \mathrm{D}$ illustration.

Skeletal reconstructions in the contemporary style of silhouette drawings are compelling and aesthetically pleasing, but they are highly simplified and may be subtly stylized. Artful restorations of missing elements incorporated in silhouette illustrations and museum mounts give an overall impression of finality and certainty. But when someone other than the artist attempts to analyze and verify the printed $2 \mathrm{D}$ reconstruction, 
the limitations of the printed medium soon become apparent Consider, for example, the mounted reconstructions and published skeletal illustrations of Brachiosaurus brancai. Once cast, or once drawn, it is a matter of some difficulty to determine where dimensional objectivity ends and subjective reconstruction begins. The posterior cervical vertebrae of this specimen were missing their neural arches. When restored and mounted, those neural arches were sculpted to be consistent with an ascending neck, with the missing zygapophyses aligned and centered as if the neck were in its undeflected state in that posture. Different illustrations, however, restore the neck with different degrees of neck elevation, and yet all depict the cervical vertebrae as essentially undeflected (JANENSCH 1950B; CZERKAS \& CZERKAS, 1991: 132, text-fig; MCINTOSH, 1997: 655, text-fig. 1; MCINTOSH, BRETT-SURMAN \& FARLOW, 1997: 286, text-fig. 20.16; WILSON \& SERENO, 1998: foldout 1). The neck elevation depicted in (MCINTOSH, 1997: 655, TEXT-FIG. 1; MCINTOSH, BRETT-SURMAN \& FARLOW, 1997: 286, text-fig. 20.16a) is achieved by drawing the centra of the cervical vertebra at the base of the neck as significantly keystoned, or trapezoidal, in profile shape. Specifically, between the anterior of D1 and the posterior of C11 one measures an angle of about $57^{\circ}$ (text-fig. 1$)^{*}$. Alternative reconstructions of B. brancai (WILSON \& SERENO, 1998: foldout 1) shows a much straighter neck, accumulating only $12^{\circ}$ from D2 to C10, and (CZERKAS \& CZERKAS, 1991: 132, text-fig), which shows only $5^{\circ}$. These latter reconstructions are more consistent with the original vertebral material (text-fig. 2), which exhibits no significant keystone shape in the centra within the cervicodor-

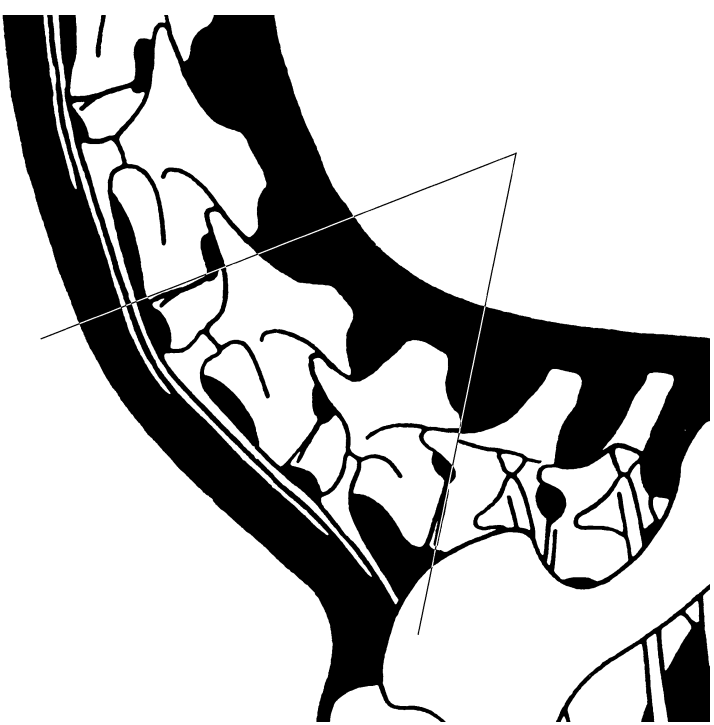

Text-fig. 2. Detail from a silhouette-style reconstruction of Brachiosaurus brancai (MCINTOSH, BRETT-SURMAN \& FARLOW, 1997: 286, text-fig. 20.16a) in which the neck achieves a vertical stance by depicting the centra of the cervical vertebra at the base of the neck as significantly keystoned, or trapezoidal, shape in lateral view. The pronounced keystone shape in this artwork is not apparent in descriptions of the original fossil material (see text-fig. 3).

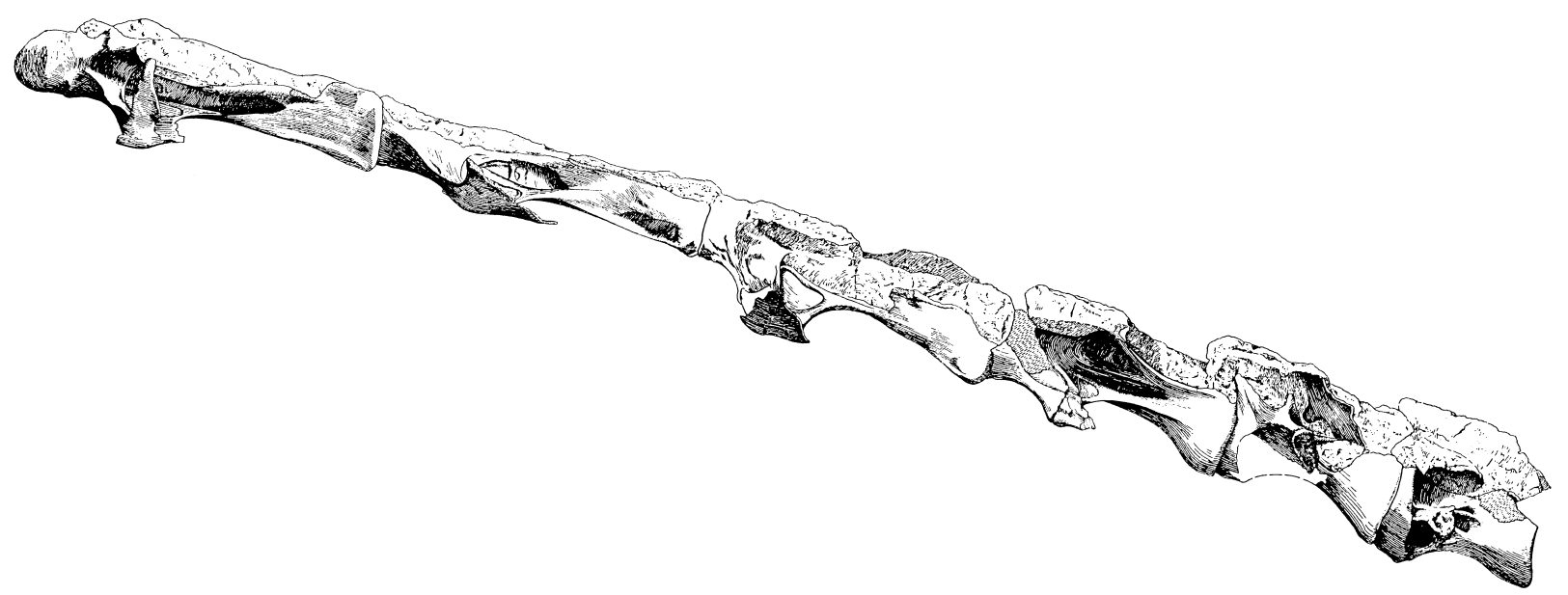

Text-fig. 3. An unretouched scan of (JANENSCH, 1950a; text-fig 49) shows the original material of Brachiosaurus brancai specimen II in the cervicodorsal region, preserved in articulation. Cervicals 10-13, and the first two dorsals share a common plane of ablation of their neural arches, however the centra are in an essentially undeflected condition, as revealed by the parallel margins of the cotyles and condyles between each successive pair of vertebrae (dorsal 1 is actually slightly dorsiflexed, as in a death pose). These six vertebrae, show that the neutral pose was straight, contrary to the popular depiction of Brachiosaurus as having a giraffe-like posture.

*) Roughly $40^{\circ}$ is due to the keystone shape of the centra of the twelfth cervical vertebra $\left(26^{\circ}\right)$ and the thirteenth cervical $\left(14^{\circ}\right)$ and the remainder presumably due to dorsiflexion. Overall, from the anterior of $\mathrm{D} 2$ to the posterior of $\mathrm{C} 10$ a total of about $68^{\circ}$ is accumulated, about $51^{\circ}$ is due the depiction of the centra in this illustration. 
Text-fig. 4. A closeup of several cervical vertebrae of Diplodocus carnegii modeled using DinoMorph $^{\mathrm{TM}}$ to study their range of motion. The zygapophyseal surfaces are based on the original specimen (Carnegie Museum of Natural History specimen CM 84), and the centra, dimensionally accurate but simplified to mere cylinders with ball-andsocket articulations.

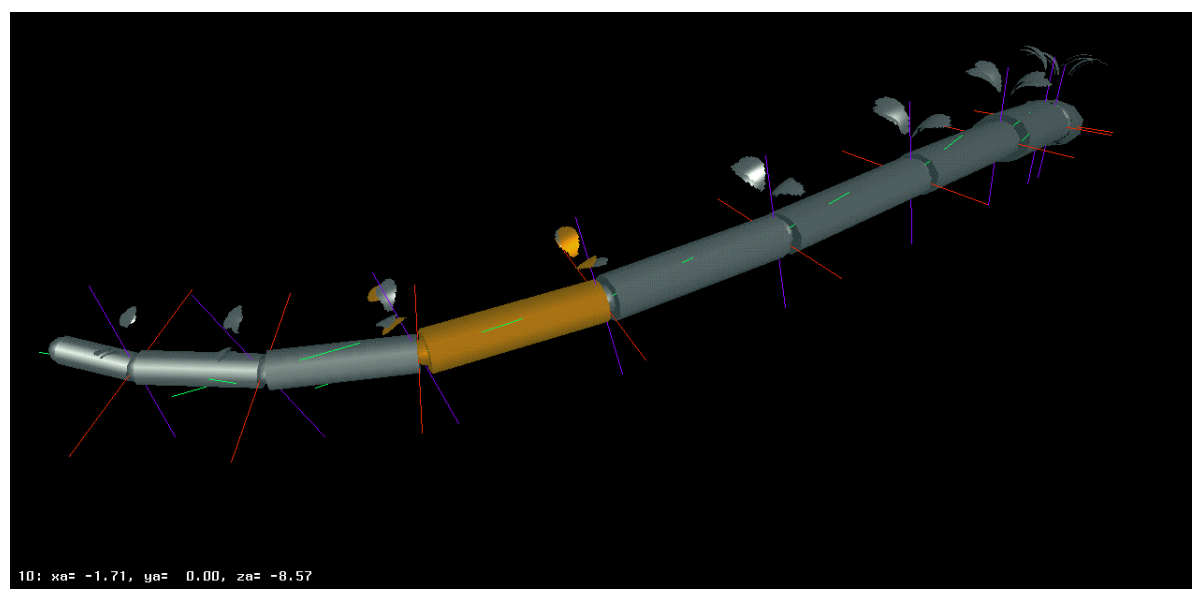

In reconstructing a skeleton from imperfect material, it is a practical necessity to introduce estimations and to make best guesses. But in seeking greater objectivity and accuracy in such reconstructions, the underlying data should be open to verification so that investigators other than the originator of the reconstruction can compare the elements of the reconstruction to the original material, and where found to be imprecise, to permit revision. These goals largely motivated DinoMorph ${ }^{\mathrm{TM}}$, a system for parametric modeling of quadrupeds, developed by the author at the University of Oregon Department of Computer and Information Science (STEVENS, 2001). Example applications of the software are described in the next section, followed by a discussion of the design behind the software.

\section{Illustrating the Parametric Approach using DinoMorph ${ }^{\mathrm{TM}}$}

In studying the range of motion of the necks of sauropod dinosaurs, their opisthocoelous cervical vertebrae were initially modeled as a series of ball-and-socket joints attached to the ends of cylinders representing the centra, with parameters including ball radius, centrum dimensions, and intervertebral separations. While the centra were highly simplified, they provided a framework on which to locate the zygapophyses and the axes of rotation. Curved surfaces that accurately replicate the individual pre- and postzygapophyses were created based on curvature measurements and tracings taken directly off the zygapophyseal facets of the original specimen (STEVENS \& PARRISH, 1999). Figure 4 shows several of the cervical vertebrae of Diplodocus in articulation; the zygapophyseal facets were scaled, oriented and positioned relative to the central condyles according to measurements from the original specimen.

To articulate these vertebrae, the axes of rotation need to be located. Fortunately, for ophistocoelous articulations, the axis for dorsoventral flexion is clearly indicated by the center of curvature of the ball-like condyle's profile in lateral view, as is the axis for mediolateral deflection indicated by the center of curvature in ventral view. But mediolateral deflection about a vertical axis resulted in the postzygapophyses contacting their associated prezygapophyses after only a few degrees of lateral deflection. Experimenting with tilting the axis anterodorsally to pass between the zygapophyses led to a more successful model for their biomechanics.

As shown in figure 5, this zygapophyseal design confers an important biomechanical advantage, for as mediolateral deflection increases the postzygapophyses rotate into progressive contact with the relatively stationary prezygapophyses, producing a gradual osteological stop at the limits of lateral deflection. The contact can occur simultaneously on opposite sides of the midline, creating a rigidly locked column of later- ally deflected vertebrae capable of resisting the torsional loads that would rise. The neck would be stabilized against the torsional loads that a wooden Chinese toy snake encounters when curled to the side. The design capitalizes on the compressive strength of the neural arches, reducing the need for ventral or dorsal bracing (MARTIN, MARTIN-ROLLAND \& FREY, 1998) to counteract axial torques. Cranial cervical vertebra in many taxa have a design similar to that in figure 5, namely steeply inclined zygapophyses that are substantially anterior to the central articulation and zygapophyseal facets that are steeply oriented. Significantly, this design is also seen in the cervical vertebrae at the base of the neck of several sauropods, including Diplodocus, Barosaurus, and Euhelopus. These taxa have very elongate centra and narrow lateral processes, and create a gracile, longer-necked form quite distinct from, say, Camarasaurus and Apatosaurus, whose massive cervicals at the base of the neck appear to employ wide-set, flat, nearly horizontal zygapophyses to achieve stabilization in lateral deflection.

To study neck pose and range of motion in the diplodocids Diplodocus carnegii and Apatosaurus louisae, simplified skeletal models were created in DinoMorph ${ }^{\mathrm{TM}}$ based on archival sources for the bone dimensions and reconstructions for the postcervical skeleton pose and body plan (STEVENS \& PARRISH, 1999). Each vertebral joint was adjusted to neutral deflection, i.e. such that the zygapophyses were centered, post- above prezygapophysis. Both necks are quite straight in neutral pose, and straight extensions of the downward sloping anterior dorsal column, resulting in their heads being held below shoulder height. With the neutral poses reconstructed, the range of motion of the neck was then explored. The limits of movement were estimated by flexing each joint up to a maximum displacement that the zygapophyses would have permitted, based on criteria for the flexibility of the synovial capsule surround- 


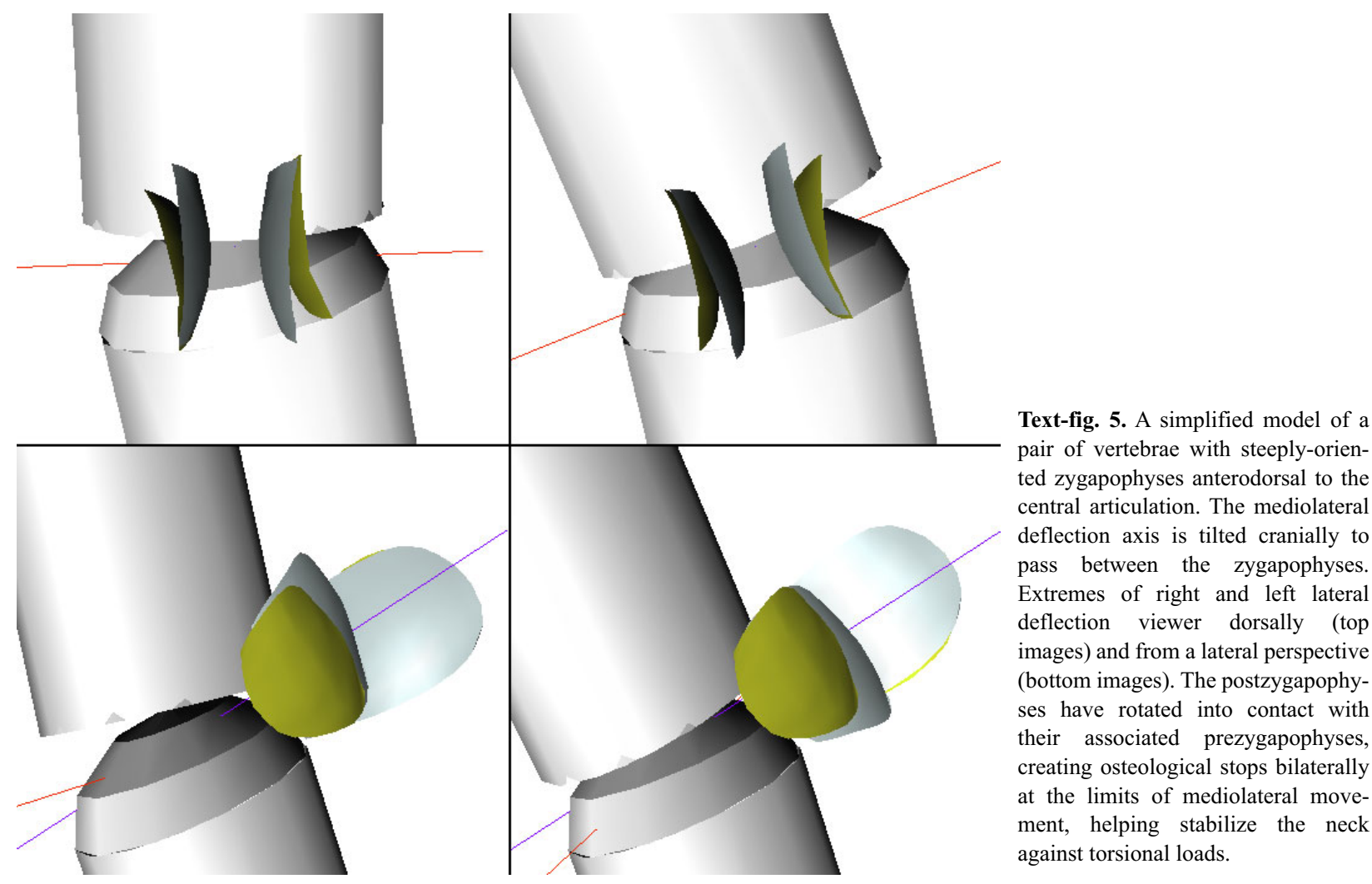

ing the zygapophyses derived from dissections of modern birds (STEVENS \& PARRISH, 1999). In figure 6 the neutral position is shown plus superimposed depictions of the neck in extremes of its dorsoventral movement for these two diplodocids. Diplodocus, despite the longer neck, showed less dorsal flexibility than Apatosaurus; both were similarly capable of remarkable ventriflexion. The body plan and flexibility of these diplodocids are consistent with low browsing, and in particular, a specialization for feeding along the shores of perennial lakes and rivers is consistent with the ability of their necks to ventriflex, their rake-like dentition, and their centers of mass placing most of the weight of the animal on to the hind limbs (STEVENS \& PARRISH, 1999).

The range of motion in these sauropod necks could be estimated with some confidence because the articular facets, particularly the zygapophyses indicated the maximum deflection achievable, beyond which the joint would approach disarticulation. While joint flexibility can be estimated along the axial skeleton with some guidance from the osteology, such is unfortunately not the case in the appendicular skeleton. Especially for long bones, the osteology may provide little evidence of the shape of the articular surfaces, creating difficulties for locating an axis of rotation. Moreover, the range of motion in the appendicular skeleton is often limited by non-osteological factors, principally ligaments (HUTCHINSON \& GATESY, 2000), which are not currently considered for inclusion into the system.
The above study incorporated a conservative model for the postcervical skeleton of Apatosaurus and Diplodocus. Head height above ground level for a given reconstruction depends on the interpretation of the trunk. Using DinoMorph ${ }^{\mathrm{TM}}$ as a mannequin, four interpretations of the trunk of Apatosaurus can be compared within a common framework. The skeletal elements are dimensionally identical in the four poses in figure 7 and correspond to A. louisae, Carnegie Museum specimen 3018. The differing trunk poses were reconstructed by viewing the digital model in left lateral aspect, with an image from one of the four illustrations superimposed (GILMORE, 1936; MCINTOSH, BRETT-SURMAN \& FARLOW; text-fig 20.12; Wilson \& SERENO, 1998; foldout 1; PlatT, 2001). The digital model was projected at the same image scale as the given illustration, positioned so their ilia were superimposed, and the hind limbs of the model were posed to superimpose over the counterparts in the illustration. Then, beginning at the sacrum and moving cranially, the curvature of the dorsal column in the digital model was adjusted, joint-by-joint, to match that in the illustration. With the two dorsal columns aligned, the scapulae and forelimb of the digital model were then adjusted to superimpose over their counterparts in the illustration. The four trunk reconstructions, published separately with similar artwork but at different scales, now share a common bone geometry. With neck and tail pose held constant, the differences specific to the trunk are readily compared. 


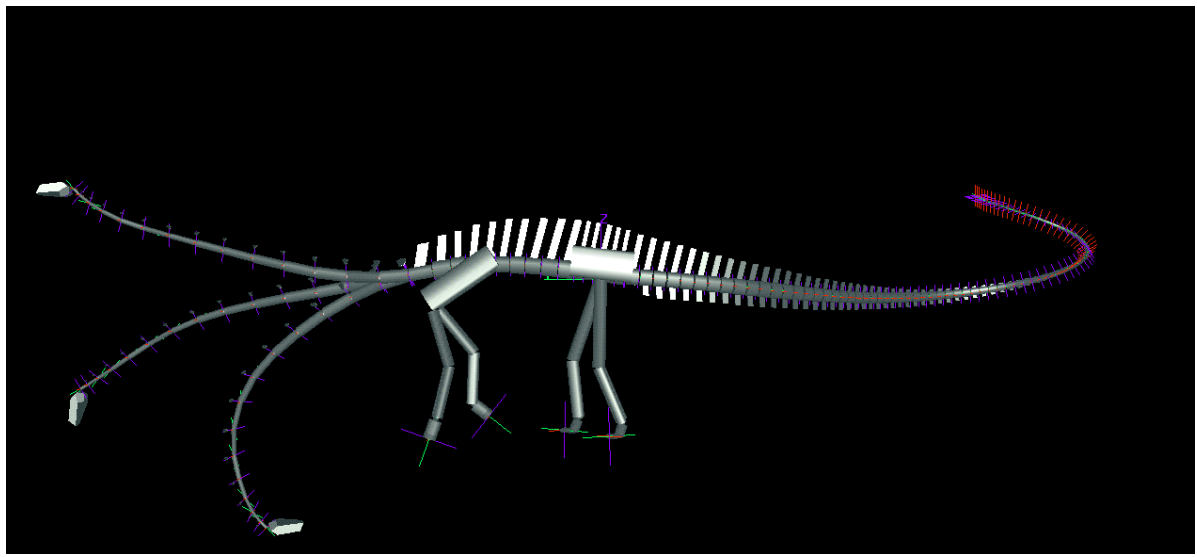

Text-fig. 6. The neutral pose plus extremes of dorsoventral flexion for Diplodocus carnegii in $a$, and for Apatosaurus louisae in $b$.

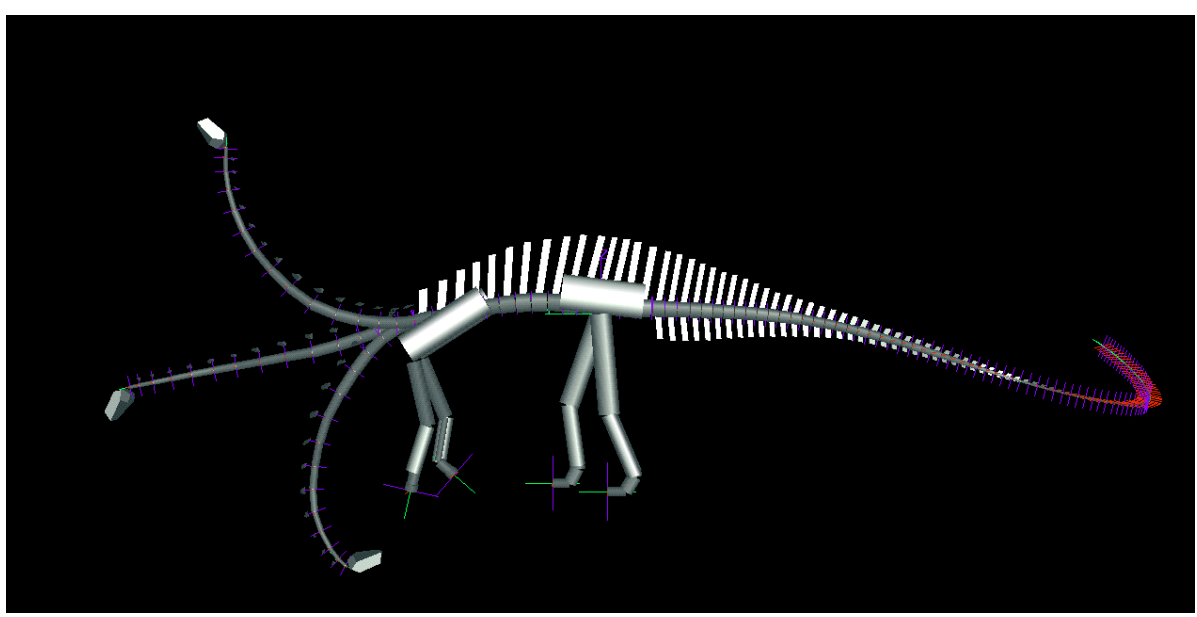

\section{Design Philosophy and Architecture}

DinoMorph ${ }^{\mathrm{TM}}$ provides user interfaces for specifying the skeletal design (phalangeal formulae, etc.), for posing and editing the geometric attributes, and for storing and sharing the data underlying the models. The software then displays the $3 \mathrm{D}$ representation within an interactive environment that is much like a conventional computer-aided design system. Originally written in the programming language $\mathrm{C}$ in 1994 , then converted to $\mathrm{C}++$ in 1996, it was originally available only on specialized graphics computers. Recently it has been rewritten in the programming language Java ${ }^{\mathrm{rM}}$ and Java $3 \mathrm{D}^{\mathrm{rM}}$ to be platform independent. As a result of five years advancement in hardware, DinoMorph $^{\mathrm{TM}}$ now presents considerably more complicated models at higher frame rates on personal computers (with 3D graphics acceleration) than the first version achieved on specialized computers that cost more than an order of magnitude more. It is being developed concurrently with new Java $3 \mathrm{D}^{\mathrm{rm}}$ releases, incorporating new features as they become available.

While the display depicts the skeleton in three dimensions it does not allow the user the natural exploratory manipu- lations and shifts of viewpoint as would naturally arise when physically handling a pair of bones to understand how they articulate. Although digital manipulation is far less vivid and immediate than holding the material in one's hands, it does provide a solution to the problem of running out of hands when attempting to articulate more than two bones at a time. Moreover, within a parametric modeling scheme these manipulations are repeatable and can be controlled precisely.

DinoMorph $^{\mathrm{TM}}$ incorporates object-oriented design and programming practices. In an object-oriented language such as $\mathrm{Java}^{\mathrm{TM}}$, classes are a means to encapsulate data and the methods (the algorithmic procedures) that operate upon that data; they specify how to create instances of a given class, and what access that object provides others to its data and methods. The remainder of this section provides an overview of the software architecture intended for general readers. In the following discussion, a Java class such as that representing a pectoral girdle, is signified by the following style and font: PectoralGirdle*.

*) Class names will occasionally be pluralized for readability, for instance saying 'Bones ' rather than a more burdensome but more technically correct phrase 'instances of class Bone'. Likewise, as in common usage, no confusion should arise in blurring the distinction between a class and instances of that class. Classes can inherit properties from other classes; a hierarchy arises when several classes share properties of a given class. They are said to extend that given class, thereby inheriting properties of the class they extend while providing additional specialization. 

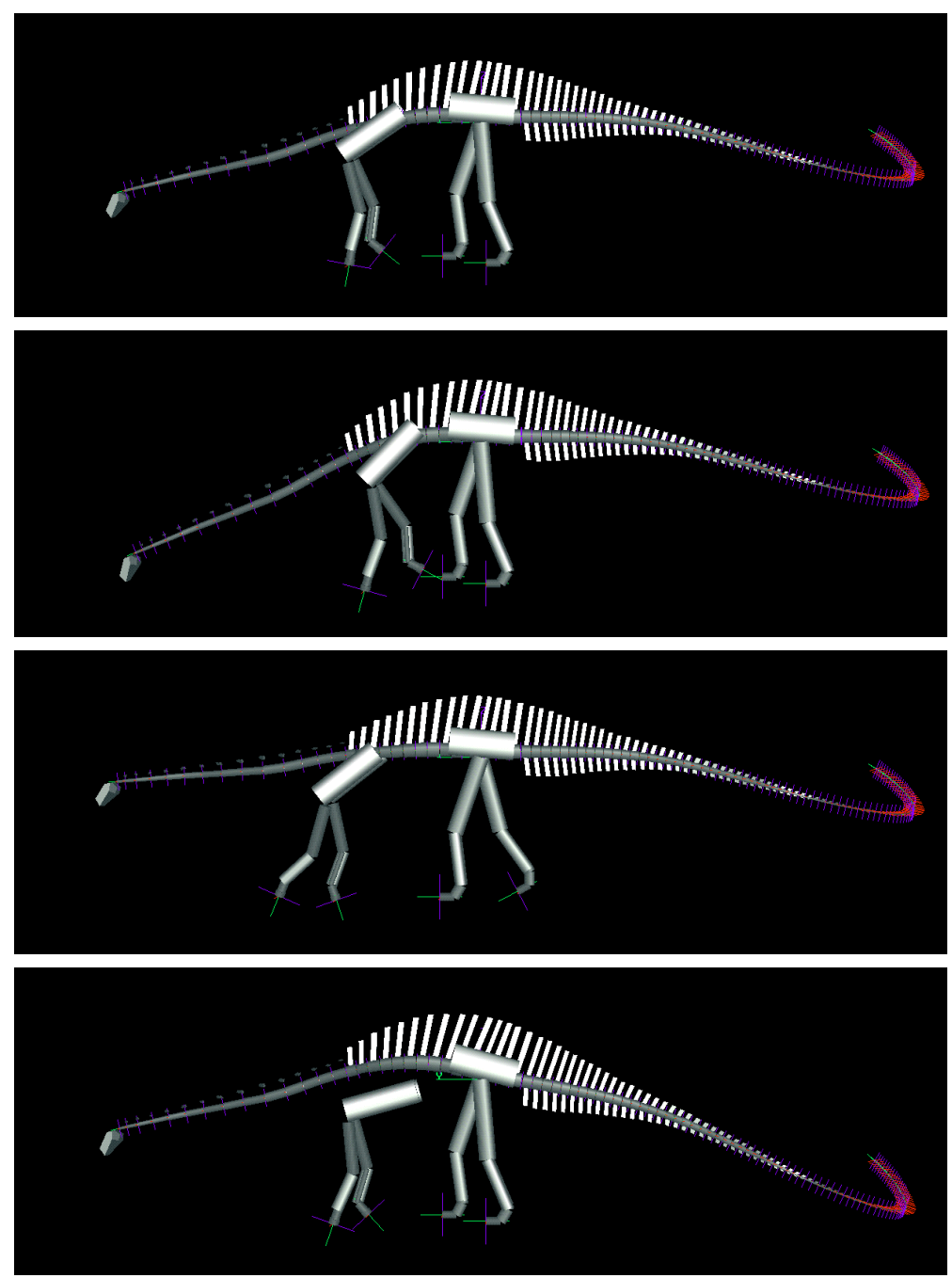

Text-fig. 7. Four interpretation of the trunk of Apatosaurus. The reconstructions in $a-d$ are based on (GILMORE, 1936; MCINTOSH, BRETT-SURMAN \& FARLOW 1997; text-fig 20.12; WILSON \& SERENO, 1998; foldout 1; PLATT, 2001) respectively. The pose of the cervical and caudal vertebrae are kept constant to emphasize the differences in the four trunk reconstructions.

\section{The Anatomy Hierarchy}

A skeleton can be thought of as a topologically-connected collection of osteological elements, articulated at some points, fused at others, and terminating in free ends at the appendages and the extremes of the vertebral column. The topology is quite uniform across quadrupeds, differing primarily in the count of vertebrae and the number of elements of the distal assemblies of the appendicular skeleton. The conventional descriptive schema in the literature reflects this framework (ROMER, 1956), and provides a hierarchy of anatomical parts and assemblies of those parts. An object-oriented design has been developed which mirrors this convention, where classes capture the different levels of structural organization and specialization implied by the common usage of the terms. With reference to figure 8 , the basic class of this hierarchy is Part (which extends the Java $3 \mathrm{D}^{\mathbf{T M}}$ class BranchGroup, as it is also a three-dimensional graphical object). In figure 8 , an arrowhead extending from some class to point to another indicates that the former is an extension, or specialization, of the latter.

Two types of Parts are distinguished: Element and Assembly. Two classes extend Element, namely Bone and Joint (a further class representing muscles is under develop- ment). Assemblies are connected collections of Parts, i.e. they may themselves contain Assemblies (e.g. the Forelimb contains the Carpus and Manus, in addition to the humerus Bone and other Elements).

Elements have a visible morphology or Shape. Just as in common usage of the terms, a Bone is not a Shape, but it has a Shape. A hierarchy of Shapes is defined within this system, from simple to complex. Shapes of differing complexity can be associated with each Element, depending on the realism and detail desired, from a simplistic cylinder to a highly complex replica of a given Bone's surface morphology. Figure 9 shows a model of Apatosaurus with a few bones rendered with some realism while the remainders are mere cylinders.

The hierarchy of Parts in figure 8 is readily extended as additional functional specialization is added. For instance, while the initial version had no representation of ribs, and only a highly schematic scapula, a detailed means to model ribs has subsequently been introduced, using Catmull-Rom splines (CATMULL \& ROM, 1974) to parametrically represent the shape of a rib, plus additional parameters specifying the position and orientation of the axis of attachment. Now curved scapular blades can lie upon a three-dimensional ribcage (see fig. 9). 


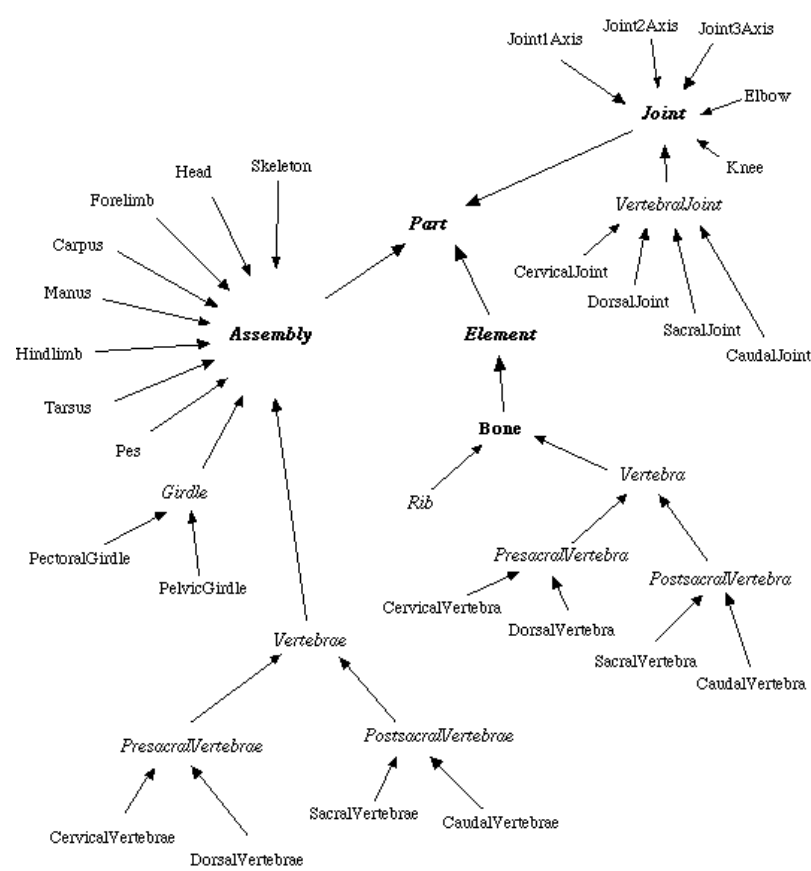

Text-fig. 8. The major classes of the anatomy hierarchy of the DinoMorph $^{\mathrm{TM}}$ design. The base class of the hierarchy is Part. Two abstract classes extend Part, namely Element and Assembly. This hierarchy shows inheritance of common properties. A DorsalVertebra extends PresacralVertebra, which is a kind of Vertebra, Bone, Element, and ultimately Part. Each level in this hierarchy adds specialization. The italicized class names correspond to abstract classes in Java ${ }^{\mathrm{TM}}$.

\section{Explicitly Representing Topology, Geometry, Pose and Morphology}

The Elements of a Skeleton have numerical parameters. Each Bone, for instance, has specified dimensions, and locations for the attachment points of the proximal and distal Joints, among its parameters. The values for these parameters are provided by a BoneSpec, one for each given Bone. Likewise there is a JointSpec specifying the parameters for each Joint, which includes the orientation and position of each component Axis. The set of BoneSpecs, JointSpecs and other types of Spec for a given model are collected together in a class called a Geometry. An instance of a Geometry specifies the values to be assumed by the parameters of the Elements of a given Skeleton.

The Elements of a Skeleton need to assume the values of the parameters stored within the Specs. This is achieved by the 'observer-observable' pattern (GAMMA, HELM, JOHNSON \& VLISSIDES, 1995), an elegant mechanism provided by $\mathrm{Java}^{\mathrm{TM}}$ by which one object can be notified when another object is changed. The Specs are 'observables", and the Elements are 'observers' of Specs. For example, if the length parameter is adjusted in the BoneSpec corresponding to the femur, that BoneSpec notifies its observers, namely the two corresponding instances Bone, which then adjust their lengths accordingly. This fundamental mechanism, by which the Skeleton assumes the values of its parameters, permits visualization of continuous Geometry changes. For example, a Skeleton can be made to dynamically 'morph' between a juvenile and adult form by interpolating the numerical parameters in its associated Geometry.

A Skeleton plus a Geometry still needs to be posed. A Pose is a set of Deflections, one for each Joint, which specifies the angles of the Joint's component Axes. To achieve the specified Pose, each Joint observes its associated Deflections (as well as its JointSpec). Modifying many Deflections in rapid succession causes the Assembly to animate like a puppet.

A Geometry plus a set of saved Poses are held in a class called a Model. Models provide methods for storing these components, for creating a specific Assembly to be visualized, for providing it with its dimensions and other parameters from its Geometry, and for providing its posture from a given Pose.

\section{Viewing and Interacting with the Model}

The user of this system views and manipulates an Assembly as if it were a three-dimensional object seen through a window. A viewer places the user at calibrated vantage point and viewing direction relative to the Assembly, and creates an image in either perspective or orthographic projection, at a specific image scale (e.g. 10:1) or an arbitrary distance, and from a specific viewpoint (e.g. dorsal) or an arbitrary perspectives. If desired, multiple Viewers can be arranged (three orthogonal plus one general viewpoint). The Assembly appears in the context of a ground plane (e.g. a $1 \mathrm{~m}$ square tile floor, or a texture-mapped pattern) and illuminated by light sources. Tools are provided to interact with the model, including measurement tools (calipers, scale bars, protractors) to interactively pose individual joints, and to adjust other parameters.

The number of adjustable parameters in DinoMorph ${ }^{\mathrm{TM}}$ is substantial. Fortunately, for the axial skeleton is possible to visualize parameters graphically, with vertebra number along the abscissa and the given parameter's value along the ordinate. The cervical, dorsal, sacral and caudal vertebrae are graphed separately since they have different vertebral counts and vary in types of parameters. The length of the caudal vertebrae, for instance, can be seen to diminish progressively with increasing vertebral position. The graph can be used as a means for entering values from an archival source or from measurements taken directly off specimens. Each axial parameter can be graphed as a pseudo-continuous mathematical spline, which greatly aids in the nonlinear interpolation of missing elements and in verifying and editing outliers that might reflect distortion or damage to the original material. The deflections along the axial skeleton are also represented in the user interface by splines. The parameters of the appendicular skeleton can be edited from menus indexed by the name of the given Bone or Joint, or by interactively clicking on the desired Element.

\section{Physics and Fidgets, Current and Future Developments}

Many biomechanical questions concern body mass, including the estimation of the total mass of an extinct animal, its hypothesized distribution in a given reconstruction, its effects on balance and dynamics, the forces required to perform movements, and so forth. Quantitative mass estimates are usually derived indirectly from the volume of three-dimensional scale 


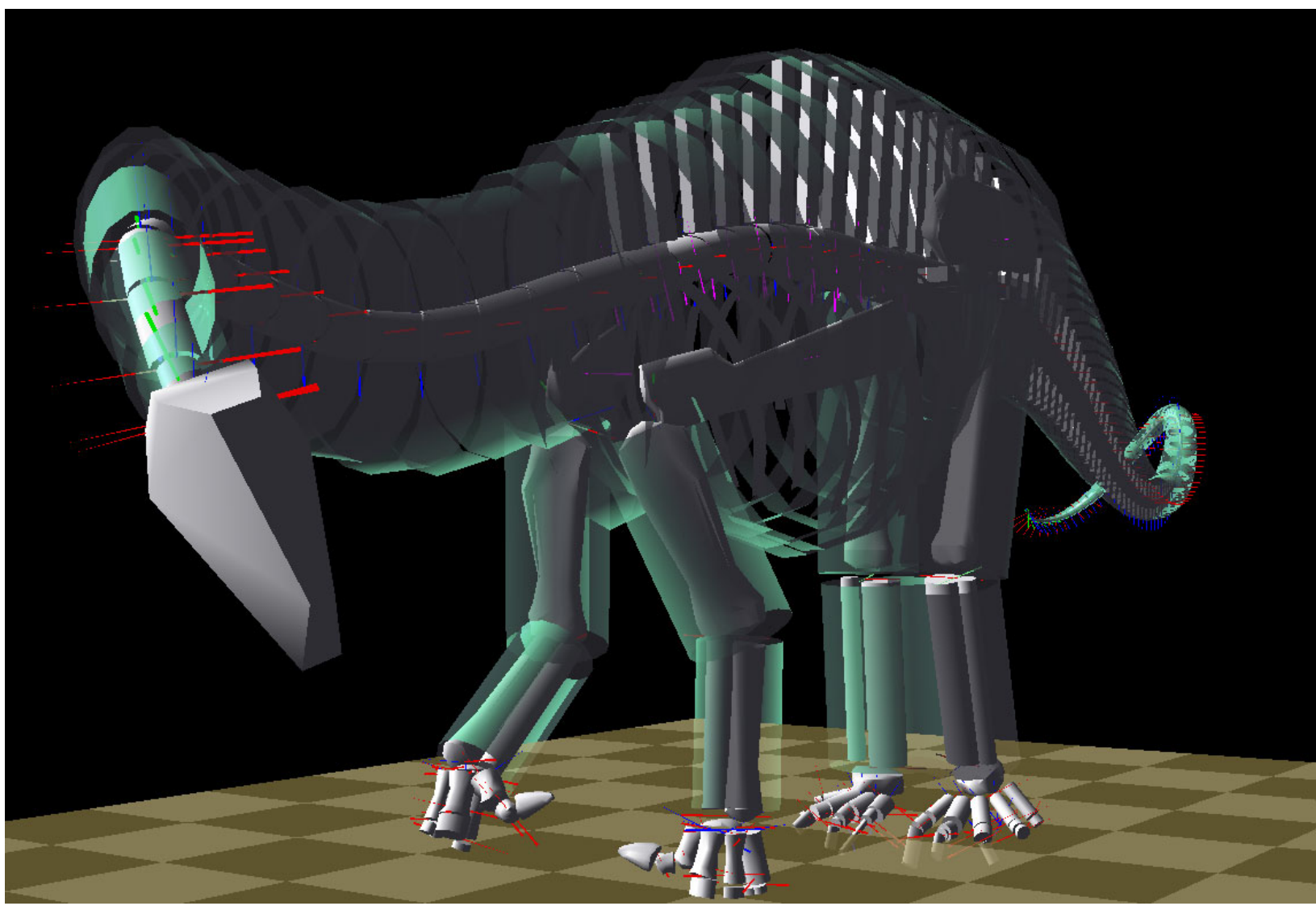

Text-fig. 9. Parametric representation of Apatosaurus louisae in DinoMorph ${ }^{\mathrm{TM}}$, showing transparent Hulls, joint axes, and a mix of cylindrical and parametrically depicted Bones.

models such as museum replicas, or digitizations thereof (ALEXANDER, 1989; HENDERSON, 1999) or from illustrations or photographs (SEEBACHER, 2001). In contrast, DinoMorph ${ }^{\mathbf{T m}}$ provides a synthetic, i.e. constructive, alternative in which the skeletal structure is directly 'fleshed out' with an incremental representation of the body volume surrounding each individual Bone. Each Bone is surrounded by an associated Hull, a cylinder of elliptical cross-section and length equal to that of the associated Bone. Parameters are provided in the BoneSpec to specify the Hull's cross-section and its position relative to the center of the Bone. Hulls (which extend Shape) are rendered as partially transparent so that the underlying skeletal structure remains visible (see text-fig. 9). By careful adjustment of the parameters of the Hulls, a smooth envelope can be constructed surrounding the Skeleton to represent an hypothesized distribution of soft tissues. The body profile defined by the Hulls can be matched with those of published life reconstructions or scale models with the advantage that this reconstruction can be posed and animated.

In addition to providing a visual representation of body bulk, the product of Hull volume and hypothesized mean density provides an estimate of the mass associated with each Bone,which can summated across the Skeleton to estimate the total mass, or used for a variety of physics-based computations. For example, the gravitational force on the neck or tail creates a torque to be counteracted, actively by muscles and passively by ligaments and so forth. The torque computation requires knowing the location of the fulcrum about which the torque is to be computed, the mass and three-dimensional location of each Element, and which Elements are distal to that fulcrum. In this example the mass associated with the head and cervical vertebrae would contribute to the suspended mass. The mathematics would be similar for a computation regarding the tail, differing primarily in being applied to a set of caudal, rather than cervical vertebrae. There is a convenient division of labor between the physics computation which can be applied to a set of Elements (and incrementally compute moments, say), and the Assembly which can provide an enumeration of its component Elements to a computation. The physics-related computations get access to these Elements through a programming technique called the 'visitor pattern' (GAMMA, HELM, JOHNSON \& VLISSIDES, 1995) wherein each Assembly is responsible for directing it to each component Element. The visitor pattern permits extensibility to the suite of physicsbased computations that might be performed on a posed Assembly.

A useful application of such mathematics is in support of posing skeletal reconstructions. Posing a whole skeleton can involve modifying hundreds of Axis angles. Just the tail of Apatosaurus alone, with its 82 vertebrae, requires the specification of 246 axis angles. The user interface provides a graphlike representation that assists in interpolating a series of Deflections along the tail, but that process would be considerably assisted by providing the tail with the appearance of 
muscular tonus and differential stiffness to bending along different axes allows naturalistic posing under the control of a few parameters that direct the tail variously. This can be approximated mathematically without introducing anatomically-based muscle models. It is just another step to provide some independent movement to the model, and for the tail to move autonomously. Such fidgeting and independent behavior, all achieved by visitors to the Assemblies, can be suppressed when undesired.

As described earlier, many factors within a skeleton are mutually constraining. Adjusting the intervertebral angles in the dorsal column requires accommodation elsewhere in the skeleton if the manus and pes are to remain in contact with the ground. Likewise, merely reposing a forelimb requires propagating postural adjustments throughout the trunk and pectoral girdles. A current development goal is the autonomous management of such adjustments, to eventually approximate the interplay between a show dog and handler: when the handler adjusts the position of a forepaw, the dog accommodates compliantly, distributing that displacement through a new posture. Approximations to this behavior would be limited to small adjustments to joint deflection well within each joint's presumed range of motion.

Another area of development is in providing DinoMorph $^{\mathrm{TM}}$ as a visualization tool for electronically published reconstructions. Programs written in $\mathrm{Java}^{\mathrm{TM}}$ can run as 'applets' on web browsers, allowing a version of DinoMorph ${ }^{\text {тм }}$ to become a visualization tool. The parametric approach becomes particularly useful in this application, for the Geometry and Pose data files are very small and readily downloaded. An internet repository of models would permit consolidation and unification of skeletal reconstructions, and also serve as an on-line resource for dimensional data, allowing both examination of the data in numerical form, and in visual, three-dimensional form.

\section{Conclusions}

DinoMorph $^{\mathrm{TM}}$ provides a parametric scheme for creating a skeletal reconstruction which offers numerous advantages over the conventional style of publishing two-dimensional reconstructions. The reconstruction is intrinsically three-dimensional, and all data is explicit and available for independent confirmation, rather than implicit in an illustration. The geometric description can be readily modified and revised and the posed changed dynamically. One is not limited to conventional views such as lateral, dorsal and anterior; a three-dimensional model can be viewed from any vantage point, with either orthographic or perspective projection. Bone morphology can be rendered at different levels of realism depending on the availability of data and the task at hand. Intermediate-detail sculpted forms are being developed, e.g. for dorsal ribs and scapulae. Parametric representations permits direct quantitative comparisons across models, parameter-by-parameter. Joint biomechanics can be studied using individually sculpted articular surfaces and models for axes of rotation. A parametric system provides an efficient means for specifying a posed, dimensionally-accurate articulated skeleton, yet the hundreds of parameters require management for the system to be practical. Tools are available for the nonlinear interpolation of parameters along the axial skeleton (both Pose and Geometry), which is particularly useful for filling in missing vertebrae and posing the hundreds of Joint axes along the vertebral column. The Elements of the trunk, however, are mutually constraining. For example changing the angular deflection at a DorsalJoint will affect the elevation of the anterior dorsal column, which must be accommodated elsewhere if the limbs are to maintain contact with the ground plane. Physically-based constraints (e.g. mass, resistance to bending) plus biologically-motivated strategies for distributing adjustments are being developed to assist in creating a compliant articulated skeletal reconstruction. Finally, an applet version of DinoMorph ${ }^{\mathrm{TM}}$ would serve as a visualizer for webbased publication of skeletal reconstructions and studies of neutral pose, range of motion, reachability, gait, growth sequences, and so forth.

\section{Acknowledgements}

Special thanks to Drs. J. MichaEl PARRISH and JAMES I. KIRKLAND for encouraging me to develop DinoMorph ${ }^{\mathrm{TM}}$, and to Mike for many subsequent discussions which helped sharpen the concept. Thanks also for the many contributions made by students in my department, including DAVID SCOTT, ZAC WOLFe, and ERIC WiLls. Per ChristiANSEN provided a very thoughtful and thorough review. Java is a registered trademark of Sun Microsystems, Inc. DinoMorph is a registered trademark of the University of Oregon.

\section{References}

Alexander, R. McN. (1989):. Dynamics of dinosaurs and other extinct giants. -Columbia New York (University Press).

Chapman, R.E., ANDERSEN, A., \& JABO, S.J. 1999 Construction of the virtual Triceratops: procedures, results and potentials. - Journal of Vertebrate Paleontology 19, suppl. pp. 37A.

CAtMull, E. \& RoM, R. (1974): A class of local interpolating Splines.- In R.Barnhill and R. Riesenfeld, [eds] Computer aided geometric design: 317-326, San Francisco (Academic Press).

Gamma, E., Helm, R., Johnson, R. \& Vlissides, J. (1995): Design Patterns, Elements of Reusable Object-Oriented Software. - Reading, Ma (Addison-Wesley).
GILlmore, C.W. (1925): A nearly complete articulated skeleton of Camarasaurus, a saurischian dinosaur from the Dinosaur National Monument. - Memoirs of the Carnegie Museum 10:347-384.

Holland, W. J. (1906): The osteology of Diplodocus Marsh. - Memoirs of the Carnegie Museum 2, 225-278.

HUTCHINSON, J.R. \& GATESY, S.M. (2000): Adductors, abductors, and the evolution of archosaur locomotion.- Paleobiology 26: 734-751.

Gatesy, S.M., Middleton, K.M., Jenkins, F.A. \& Shubin, N.H. (1999): Three-dimensional preservation of movements in Triassic theropod dinosaurs. - Nature 399, 13 May, pp. 141-144.

GILLMORE, C.W. (1925): A nearly complete articulated skeleton of Camarasaurus, a saurischian dinosaur from the Dinosaur National Monument. - Memoirs of the Carnegie Museum 10:347-384. 
Gillmore, C.W. (1936): The osteology of Apatosaurus with special reference to specimens in the Carnegie Museum. - Memoirs of the Carnegie Museum 11:175-300.

HENDERSON, D.M. (1999): Estimating the masses and centers of mass of extinct animals by 3 -D mathematical slicing. - Paleobiology 25:88-106.

JANENSCH, W. (1950): Die Wirbelsäule von Brachiosaurus brancai.Palaeontographica (suppl. 7) 3: 27-93. 1950a.

JANENSCH, W. (1950): Die Skelettrekonstrucktion von von Brachiosaurus brancai. - Palaeontographica (suppl. 7) 3: 97-102. 1950b.

Martin, J., V. MARTIN-Rolland \& FREY, E. (1998): Not cranes or masts, but beams: The biomechanics of sauropod necks. Oryctos 1:113-120.

McIntosh, J. (1997): Sauropoda. - In P.J. Currie and K. Padian [eds] Encyclopedia of dinosaurs: pp. 654- 658. San Diego (Academic Press).

McIntosh, J., M.K. Brett-Surman \& FARLOW, J. O. (1997): Sauropods. - In J.O. Farlow and M.K. Brett-Surman [eds], The complete dinosaur: pp. 264-290. Bloomington (Indiana University Press).

Osborn, H.F. \& Mook, C.C. (1921): Camarasaurus, Amphicoelias, and other sauropods of Cope. - Memoirs of the American Museum of Natural History. 3:247-287.
PAul, G.S. \& Christiansen, P. (2000) Forelimb posture in neoceratopsian dinosaurs: implications for gait and locomotion. - Paleobiology 26: 450-465

Platt, P. (2001): The pectoral girdle of the sauropod dinosaur Apatosaurus. - Bulletin of the Mesa Southwest Museum 8, in press.

ROMER, A.S. (1956): Osteology of the Reptiles. - Chicago (University of Chicago Press) 772 pp.

SEEBACHER, F. (2001): A New method to calculate allometric lengthmass relationships of dinosaurs. - Journal of Vertebrate Paleontology 21:51-60.

STEVENS, K.A. \& PARRISH, J.M. (1999): Neck posture and feeding habits of two Jurassic sauropod dinosaurs. - Science, April 30. 284:798-800.

STEVENS, K.A (2000): http://www.cs.uoregon.edu/ kent/ DinoMorph.html.

WALTERS, R.F., CHAPMAN, R.E, SNYDER, R.A., \& MOHN, B.J. (2000): Using virtual skeletons as a basis for reconstructing fossil vertebrates. - Journal of Vertebrae Paleontology 20, suppl. pp. 76A.

WILSON, J.A. \& SERENO, P.C. (1998): Early evolution and higher-level phylogeny of sauropod dinosaurs. - Society of Vertebrate Paleontology Memoir 5:1-68. 